\title{
Weak and Ultrastrong Coupling Limits of the Quantum Mean Force Gibbs State
}

\author{
J. D. Cresser( $\oplus^{1,2,3, *}$ and J. Anders $\oplus^{1,4, \dagger}$ \\ ${ }^{1}$ Department of Physics and Astronomy, University of Exeter, Stocker Road, Exeter EX4 4QL, United Kingdom \\ ${ }^{2}$ School of Physics and Astronomy, University of Glasgow, Glasgow G12 8QQ, United Kingdom \\ ${ }^{3}$ Department of Physics and Astronomy, Macquarie University, 2109 New South Wales, Australia \\ ${ }^{4}$ Institut für Physik und Astronomie, University of Potsdam, 14476 Potsdam, Germany
}

(Received 30 April 2021; accepted 11 October 2021; published 17 December 2021)

\begin{abstract}
The Gibbs state is widely taken to be the equilibrium state of a system in contact with an environment at temperature $T$. However, non-negligible interactions between system and environment can give rise to an altered state. Here, we derive general expressions for this mean force Gibbs state, valid for any system that interacts with a bosonic reservoir. First, we derive the state in the weak coupling limit and find that, in general, it maintains coherences with respect to the bare system Hamiltonian. Second, we develop a new expansion method suited to investigate the ultrastrong coupling regime. This allows us to derive the explicit form for the mean force Gibbs state, and we find that it becomes diagonal in the basis set by the systemreservoir interaction instead of the system Hamiltonian. Several examples are discussed including a single qubit, a three-level $V$-system, and two coupled qubits all interacting with bosonic reservoirs. The results shed light on the presence of coherences in the strong coupling regime, and provide key tools for nanoscale thermodynamics investigations.
\end{abstract}

DOI: 10.1103/PhysRevLett.127.250601

The last decade has seen much progress in building a comprehensive framework of "strong coupling thermodynamics" [1] that extends standard thermodynamic relations to take into account the impact of system-environment interactions [2-23]. Based on the formal concept of the mean force Gibbs (MFG) state [1,24], strong coupling thermodynamic potentials have been identified $[8-11,14]$, detailed entropy fluctuation relations have been shown to hold [12,13], and quantum measurements have been included in a stochastic description of strongly coupled quantum systems [18]. In quantum thermometry, strong coupling has been found to improve measurement precision $[19,20]$, while it can be detrimental for the efficiency of quantum engines [21].

For classical nanoscale systems, the impact of the environment, or reservoir, beyond setting the system's temperature has been known since the 1930s [24]. System-reservoir interactions lead to a modification of the system's bare Hamiltonian $H_{S}$ to an effective (mean force) Hamiltonian which is routinely calculated for classical systems in chemistry simulations [25]. The resulting classical distribution or quantum state of the system, known as the MFG state, is the reduced state

$$
\rho_{S}=\operatorname{Tr}_{R}\left[\tau_{S R}(\beta)\right]=\operatorname{Tr}_{R}\left[\frac{e^{-\beta H_{S R}}}{Z}\right]
$$

of the global system-plus-reservoir Gibbs state $\tau_{S R}(\beta)$ at inverse temperature $\beta=1 / k_{B} T$. The predictions of strong coupling thermodynamics differ from standard thermodynamics because the MFG state can deviate significantly from the standard Gibbs state $\tau_{S}(\beta) \propto e^{-\beta H_{S}}$ widely used across all of the natural sciences. Apart from leading to corrections to the state's probabilities, the systemreservoir coupling can lead to $\rho_{S}$ maintaining coherences with respect to the basis of $H_{S}$ at low and intermediate temperatures [26]. This is significant because coherences are often viewed as an indication of the quantumness of a system and considered a quantum "resource" [27]. Beyond quantum thermodynamics [26,28-32], coherences play an important role in some biological processes [33-37], and may also affect a material's magnetization behavior [38].

But beyond a few limited examples, the explicit evaluation of the reduced state $\rho_{S}$ has generally proven intractable. This lack of immediately applicable expressions of $\rho_{S}$ severely hampers progress in applying strong coupling thermodynamics methods to concrete systems, as well as characterizing thermodynamic properties of strongly coupled equilibrium states, including the presence of coherences.

Here, we provide explicit forms of the MFG state for general quantum systems coupled to bosonic environments, in the weak and ultrastrong coupling limit, respectively. For the previously unexplored ultrastrong coupling regime, we develop a new perturbative approach which leads to a surprisingly neat expression for the MFG state. For the weak coupling limit we give concrete conditions on the coupling strength $\lambda$ for the coupling to be considered "weak." Several representative examples are discussed in both regimes, including a single qubit, a three-level 
$V$-system, and two coupled qubits all interacting with bosonic reservoirs.

General setting.-Throughout, we consider global equilibrium states $\tau_{S R}(\beta) \propto e^{-\beta H_{S R}}$ of a system $S$ coupled to bosonic reservoir(s) $R$. For a single continuous reservoir, the full Hamiltonian $H_{S R}$ is $[39,40]$

$H_{S R}=H_{S}+\int_{0}^{\infty} d \omega \frac{p^{2}(\omega)+\left(\omega q(\omega)+\lambda \sqrt{\frac{2 J(\omega)}{\omega} X}\right)^{2}}{2}$,

where $\left[q(\omega), p\left(\omega^{\prime}\right)\right]=i \delta\left(\omega-\omega^{\prime}\right)$ are the commutation relations for reservoir position and momentum operators [41]. We choose units with $\hbar=1$ and $k_{B}=1$ throughout. The strength of the system-reservoir coupling is scaled by a dimensionless factor $\lambda$, where the coupling is to an arbitrary dimensionless system operator $X=X^{\dagger}$, and $J(\omega)$ is a real function of $\omega$ which will later be identified as the reservoir spectral density. An extension of (2) to two reservoirs will also be considered.

While for $\lambda \rightarrow 0$ the MFG state $\rho_{S}$ clearly reduces to $\tau_{S}(\beta)=e^{-\beta H_{S}} / Z_{S}$ with $Z_{S}=\operatorname{Tr}\left[e^{-\beta H_{S}}\right]$, it will differ considerably from $\tau_{S}$ for non-negligible $\lambda$. Here, we solve this problem for a number of physically meaningful cases. To prepare the derivation, we expand $H_{S R}=H_{S}^{\prime}+$ $H_{R}+\lambda V$ as a sum of a reservoir Hamiltonian $H_{R}=\int_{0}^{\infty} d \omega \omega\left[b^{\dagger}(\omega) b(\omega)+b(\omega) b^{\dagger}(\omega)\right] / 2$ with $b(\omega)=$ $\sqrt{\omega / 2}[q(\omega)+i p(\omega) / \omega]$ reservoir annihilation operators, an interaction energy $\lambda V=\lambda X B$ with $B=\int_{0}^{\infty} d \omega \sqrt{J(\omega)} \times$ $\left[b(\omega)+b^{\dagger}(\omega)\right]$, and an effective system Hamiltonian $H_{S}^{\prime}=H_{S}+\lambda^{2} X^{2} Q$. Here, $Q=\int_{0}^{\infty} d \omega J(\omega) / \omega$ is the reorganization energy [43-45]. The latter has a nontrivial impact on $\rho_{S}$ only if $X^{2} \propto \mathbb{I}$. Thus for many qubit problems, for which $X \sim \sigma_{\hat{\mathbf{r}}}$ for some Pauli-matrix $\sigma_{\hat{\mathbf{r}}}$, the reorganization energy can be disregarded as a constant offset. Before discussing the different coupling limits, we first comment on the high temperature limit at all finite coupling strengths.

High temperature limit.-For $\beta \rightarrow 0$ the trace over the reservoir in $\rho_{S}=\operatorname{Tr}_{R}\left[\tau_{S R}(\beta)\right]$ can be performed directly using a factorization approximation of $\exp \left[-\beta H_{S R}\right]$, see the Supplemental Material [46]. One finds the cancellation of the reorganization energy term in $H_{S}^{\prime}$, yielding $\rho_{S}=$ $\tau_{S}(\beta)+\mathcal{O}\left(\beta^{2}\right)$, i.e., the system's Gibbs state with respect to the bare Hamiltonian $H_{S}$ emerges.

Weak coupling.-We now turn to arbitrary temperatures and consider the weak coupling limit, quantitatively defined in Eq. (5) below. To obtain $\rho_{S}$, we write the system operator $X$ in terms of the energy eigenoperators $X_{n}$ for the system, i.e., $X=\sum_{n} X_{n}$ where $n$ ranges over positive and negative values. The $X_{n}$ are defined through $\left[H_{S}, X_{n}\right]=$ $\omega_{n} X_{n}$ with $\omega_{n}$ the Bohr frequencies. Since $X=X^{\dagger}$ one has $X_{n}=X_{-n}^{\dagger}$ and $\omega_{n}=-\omega_{-n}$, with $\omega_{0}=0$. Using the Kubo expansion we obtain the MFG state, correct to second order in coupling $\lambda[26,52-55]$ indicated by the superscript ${ }^{(2)}$,

$$
\begin{aligned}
\rho_{S}^{(2)}= & \tau_{S}+\lambda^{2} \beta \sum_{n} \tau_{S}\left(X_{n} X_{n}^{\dagger}-\operatorname{Tr}_{S}\left[\tau_{S} X_{n} X_{n}^{\dagger}\right]\right) \mathcal{D}_{\beta}\left(\omega_{n}\right) \\
& +\lambda^{2} \sum_{n}\left[X_{n}^{\dagger}, \tau_{S} X_{n}\right] \frac{d \mathcal{D}_{\beta}\left(\omega_{n}\right)}{d \omega_{n}} \\
& +\lambda^{2} \sum_{m \neq n}\left(\left[X_{m}, X_{n}^{\dagger} \tau_{S}\right]+\text { H.c. }\right) \frac{\mathcal{D}_{\beta}\left(\omega_{n}\right)}{\omega_{m n}},
\end{aligned}
$$

where $\omega_{m n}=\omega_{m}-\omega_{n}$ are frequency differences, the double sum is over all ordered pairs $(m, n)$ with $m \neq n$, and derivation details are given in the Supplemental Material [46]. The temperature dependent coefficient $\mathcal{D}_{\beta}\left(\omega_{n}\right)$ includes generally principal part integral transforms of reservoir correlation functions involving $J(\omega)$ and Bose number statistics $n_{\beta}(\omega)$. These integrals are responsible for population-coherence coupling, terms that are routinely ignored in the Bloch-Redfield master equation description of the dynamics, in which case the steady state simplifies to $\tau_{S}$ [56]. The impact of these terms here is that the reduced state (3) can differ very significantly from $\tau_{S}$. In particular, $\rho_{S}^{(2)}$ may maintain energetic coherences (coherences in the basis of the bare Hamiltonian $H_{S}$ ) since the commutator

$$
\left[\rho_{S}^{(2)}, H_{S}\right]=\lambda^{2} \sum_{m \neq n}\left(\left[X_{m}, X_{n}^{\dagger} \tau_{S}\right]+\text { H.c. }\right) \mathcal{D}_{\beta}\left(\omega_{n}\right),
$$

is in general nontrivial.

We highlight that the derivation of (3) requires

$$
|\lambda| \ll \frac{1}{\sqrt{\left|\beta \sum_{n} \operatorname{Tr}_{S}\left[\tau_{S} X_{n} X_{n}^{\dagger}\right] \mathcal{D}_{\beta}\left(\omega_{n}\right)\right|}}
$$

to be valid [46]. Beyond the loose requirement that $\lambda$ ought to be "small," this condition gives a well-quantified limit for $\lambda$ being in the weak coupling regime at a given $\beta$. Note that the range of $\lambda$ for which the weak coupling regime and hence (3) is applicable changes as a function of temperature, with larger temperature generally allowing larger $\lambda$.

As a first example for expression (3) we consider the spinboson model, i.e., a single qubit with Hamiltonian $H_{S}=\omega_{q} \sigma_{z} / 2$, coherently coupled to a bosonic bath with $X=\sigma_{z} \cos \theta-\sigma_{x} \sin \theta$. This model describes a charge qubit in a double quantum dot [26], as well as the stochastic behavior of spins in magnetic materials [38]. Coherences have recently been identified in the MFG state for this example [26], and our expression (3) reproduces these results [46].

As a novel illustration of the power of (3) we consider the three-level $V$-system with Hamiltonian $H_{S}=0|0\rangle\langle 0|+$ $\omega_{1}|1\rangle\left\langle 1\left|+\omega_{2}\right| 2\right\rangle\langle 2|$ with $\omega_{1,2}=\omega_{q} \mp \Delta / 2$ and $\Delta / 2 \ll \omega_{q}$, coupled to the reservoir via $X=\sqrt{2}|\psi\rangle\langle 0|+$ H.c., where $|\psi\rangle=(|1\rangle+|2\rangle) / \sqrt{2}$. These systems can represent biomolecules and have attracted significant attention as their dynamics, according to a Bloch-Redfield master equation, 
gives rise to metastable noise-induced energetic coherences [57]. These dynamical coherences are long lived, but they eventually decay. However, the Bloch-Redfield approach makes approximations which, despite non-negligible environment coupling, force $\tau_{S}$ to be the steady state from the outset. In contrast, when the reservoir impact is included in the form of the MFG state $\rho_{S}^{(2)}$, energetic coherences persist in the $V$-system even in equilibrium.

To obtain $\rho_{S}^{(2)}$ we identify the eigenoperators and Bohr frequencies $\left(X_{n}, \omega_{n}\right)$ as $\left(|1\rangle\langle 0|, \omega_{1}\right),\left(|2\rangle\langle 0|, \omega_{2}\right)$, $\left(|0\rangle\langle 2|,-\omega_{2}\right)$, and $\left(|0\rangle\langle 1|,-\omega_{1}\right)$. Substituted into (3) one obtains

$\rho_{S}^{(2)}=\tau_{S}+\lambda^{2} \sum_{p=0,1,2} f_{p}(\beta)|p\rangle\langle p|+\lambda^{2} g(\beta)(|1\rangle\langle 2|+| 2\rangle\langle 1|)$.

Expressions for $\lambda^{2} g(\beta)$, as well as the diagonal coupling corrections $\lambda^{2} f_{p}(\beta)$ are given in Eq. (SM52) in [46], and plotted as a function of temperature $T$ in Fig. 1. Of particular significance is the presence of nonvanishing coherence $g(\beta)$ between the upper levels $|1\rangle$ and $|2\rangle$. At low temperatures coherence arises due to the environment's vacuum fluctuations, while it depletes at higher temperature due to classical fluctuations. Coherence in fact peaks at an intermediate temperature whose scale is set by $\omega_{q}$, as confirmed numerically.

Ultrastrong coupling.-To derive the MFG state $\rho_{S}$ in the ultrastrong coupling limit $\lambda \rightarrow \infty$, perturbative techniques following those used at weak system-reservoir coupling are inapplicable and an entirely new approach is required. We here develop such a new approach [46], by reversing the roles of system and perturbation via $H_{S}^{\prime}+H_{R}+\lambda V \rightarrow \lambda\left[V+\lambda^{-1}\left(H_{S}^{\prime}+H_{R}\right)\right]$. Building on techniques previously used to study unitary Zeno dynamics [58-61], we here apply it in a very different context: in the temperature domain and generalized to open systems which requires carrying out a highly nontrivial trace over the reservoir. Importantly, we show that all diverging terms with positive powers of $\lambda$ cancel. Equipped with our ultrastrong expansion method one finds, for any quantum system coupled to a bosonic reservoir, the MFG state

$$
\lim _{\lambda \rightarrow \infty} \rho_{S}=\frac{e^{-\beta \sum_{n} P_{n} H_{S} P_{n}}}{\operatorname{Tr}\left[e^{-\beta \sum_{n} P_{n} H_{S} P_{n}}\right]},
$$

where $P_{n}=\left|x_{n}\right\rangle\left\langle x_{n}\right|$ are projection operators on the nondegenerate eigenstates $\left|x_{n}\right\rangle$ of $X$. This general analytical form of the MFG state in the ultrastrong limit is the main finding of this Letter. To our knowledge, it constitutes a completely new result not previously suggested or proven in the literature, not even for specific systems.

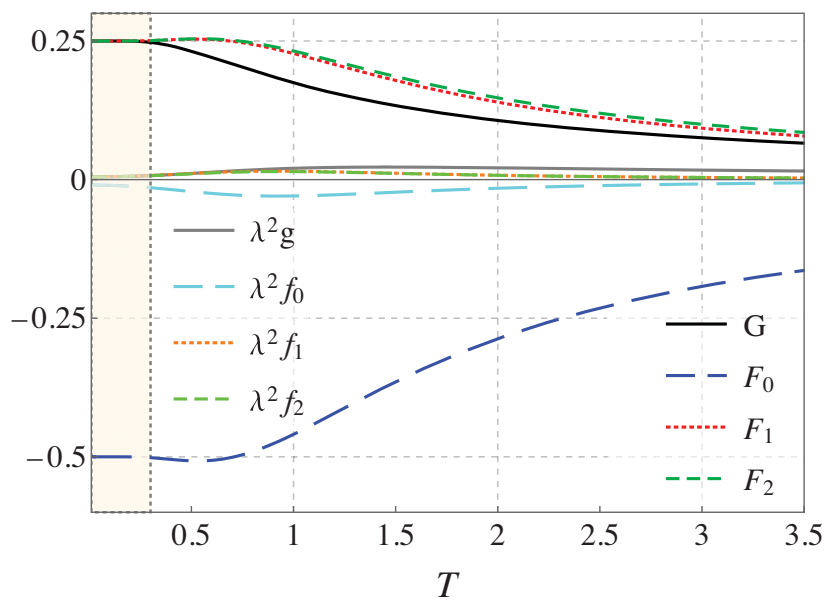

FIG. 1. Mean force Gibbs state corrections for the $V$-system as a function of temperature $T$. In the weak coupling limit, with $\lambda=0.1$, the $|1\rangle\langle 2|$ coherence is $\lambda^{2} g$ (solid gray) and the population corrections are $\lambda^{2} f_{p}$ for the three energy eigenstates $p=0,1,2$ (cyan, large-dashed; orange, dotted; light green, dashed), see Eq. (6). The weak coupling result is valid in the white-shaded temperature range, where condition (5) is obeyed. In the ultrastrong coupling limit, $\lim _{\lambda \rightarrow \infty}$, the MFG state $\rho_{S}$ is a specific case of (7), and given in Eq. (SM102) in [46]. Its coherence is $G=\lim _{\lambda \rightarrow \infty}\left\langle 1\left|\rho_{S}\right| 2\right\rangle+$ H.c. (solid black) and the population corrections are $F_{p}=\lim _{\lambda \rightarrow \infty}\left\langle p\left|\rho_{S}\right| p\right\rangle-\left\langle p\left|\tau_{S}\right| p\right\rangle$ (blue, large-dashed; red, dotted; green, dashed), showing evidence of significant deviations of $\rho_{S}$ from $\tau_{S}$. Parameters for this plot: $\omega_{q}=3$ and $\Delta=0.1$, and the environment spectral density is $J(\omega)=Q \tau_{c} \omega e^{-\tau_{c} \omega}$ with $Q=10$ and $\tau_{c}=1$.

The interpretation of the MFG state in (7) is that it is still a Gibbs state, but with respect to an effective Hamiltonian $\sum_{n} P_{n} H_{S} P_{n}$. The impact of ultrastrong coupling is to force the system to equilibrate according to the eigenstates $P_{n}$ of the now dominant system interaction operator $X$, rather than with respect to the system Hamiltonian $H_{S}$. Analogously to the standard Gibbs state $\tau_{S} \propto e^{-\beta H_{S}}=$ $\sum_{k} e^{-\beta E_{k}}\left|e_{k}\right\rangle\left\langle e_{k}\right|$ where the weights in the exponents are given by the mean value $E_{k}=\left\langle e_{k}\left|H_{S}\right| e_{k}\right\rangle$ of the system Hamiltonian in the energy eigenbasis $\left|e_{k}\right\rangle$, the ultrastrong MFG state has weights in the exponent that are given by the mean value of the effective Hamiltonian in the effective eigenbasis $\left|x_{n}\right\rangle$, i.e., $\left\langle x_{n}\left|\sum_{m} P_{m} H_{S} P_{m}\right| x_{n}\right\rangle=\left\langle x_{n}\left|H_{S}\right| x_{n}\right\rangle$. While the derivation of (7) assumes nondegenerate eigenstates $\left|x_{n}\right\rangle$ [62], extensions to degenerate eigenspaces are straightforward.

We note that Kawai and co-workers have recently conjectured the form of the system's dynamical state at long times [63,64], i.e., the steady state $\varrho_{S}^{s S}(t \rightarrow \infty)$, to be $\varrho_{S}^{\text {conj }}=\sum_{n} P_{n} \tau_{S} P_{n}$. This form looks similar to our derived MFG state $\rho_{S}$ in (7) but is structurally different, as exemplified below. 
As an illustration of Eq. (7) we consider again the single qubit, now ultrastrongly coupled to a reservoir with $X=\boldsymbol{\sigma} \cdot \hat{\mathbf{r}}=\sigma_{\hat{\mathbf{r}}}$ with $\hat{\mathbf{r}}$ an arbitrary unit vector. With the projectors on the eigenstates of $\sigma_{\hat{\mathbf{r}}}, P_{0}=\left|+_{\hat{\mathbf{r}}}\right\rangle\left\langle+_{\hat{\mathbf{r}}}\right|$ and $P_{1}=\left|{ }_{\hat{\mathbf{r}}}\right\rangle\left\langle{ }_{\hat{\mathbf{r}}}\right|$, the partitioned Hamiltonian becomes $\sum_{n} P_{n} H_{S} P_{n}=\sigma_{\hat{\mathbf{r}}} \cos (\theta) \omega_{q} / 2$ where $\hat{\mathbf{r}} \cdot \hat{\mathbf{z}}=\cos (\theta)$. A straightforward calculation then gives the MFG state [46],

$$
\lim _{\lambda \rightarrow \infty} \rho_{S}=\frac{1}{2}\left[1-\sigma_{\hat{\mathbf{r}}} \tanh \left(\frac{1}{2} \beta \omega_{q} \cos (\theta)\right)\right] .
$$

Being diagonal in the basis of the coupling-operator $X$, for $\cos (\theta) \neq 0$ this state clearly maintains coherences with respect to the Hamiltonian's $\sigma_{z}$ basis.

For comparison we note that the conjectured steady state for this system is $\varrho_{S}^{\text {conj }}=\frac{1}{2}\left[1-\sigma_{\hat{\mathbf{r}}} \cos (\theta) \tanh \left(\frac{1}{2} \beta \omega_{q}\right)\right]$ [64], which differs from (8) in the positioning of $\cos (\theta)$. Evidence, based on numerically solving hierarchical equations of motion (HEOM), that the dynamical steady state $\varrho_{S}^{s S}$ is numerically close to $\varrho_{S}^{\text {conj }}$ has been provided [64]. However, for the specific inverse temperature used in the numerics, the difference between $\varrho_{S}^{\text {conj }}$ and the MFG state (8) is negligible, and hence the convergence could equally be to (8) instead [46].

A second example of the application of Eq. (7) is the three-level $V$-system for which the ultrastrong coupling MFG state is given in Eq. (SM102) in [46]. Its deviations from $\tau_{S}$ are plotted in Fig. 1, together with the weak coupling corrections given in (6). Coherence between the excited states, $|1\rangle$ and $|2\rangle$, is found to persist at low and intermediate temperatures $T \lesssim \omega_{q}$. As expected, the corrections in the ultrastrong limit are larger in magnitude than those at weak coupling.

The ultrastrong coupling derivation resulting in Eq. (7) can further be extended to situations involving two reservoirs, both at the same inverse temperature $\beta$. Here, we consider two systems $S_{1}$ and $S_{2}$ (such as qubits), that interact with each other via $H_{12}^{\text {int }}$ as well as each coupling to a bath, through $\lambda_{1} B_{1} X_{1}$ and $\lambda_{2} B_{2} X_{2}$, respectively. This gives the total Hamiltonian $H_{\lambda_{1} \lambda_{2}}=H_{S}^{\prime}+$ $\lambda_{1} B_{1} X_{1}+\lambda_{2} B_{2} X_{2}+H_{B}$ with two-system Hamiltonian $H_{S}^{\prime}=H_{1}+H_{2}+H_{12}^{\text {int }}+\lambda_{1}^{2} X_{1}^{2} Q_{1}+\lambda_{2}^{2} X_{2}^{2} Q_{2}$, and tworeservoir Hamiltonian $H_{B}=H_{B 1}+H_{B 2}$. At ultrastrong coupling we find the MFG state of the combined system $S$ to be [46],

$$
\rho_{S}=\frac{e^{-\beta \sum_{m n} P_{1 m} \otimes P_{2 n} H_{S} P_{1 m} \otimes P_{2 n}}}{\operatorname{Tr}\left[e^{-\beta \sum_{m^{\prime} n^{\prime}} P_{1 m^{\prime}} \otimes P_{2 n^{\prime}} H_{S} P_{1 m^{\prime}} \otimes P_{2 n^{\prime}}}\right]},
$$

where $P_{\alpha n}=\left|x_{\alpha n}\right\rangle\left\langle x_{\alpha n}\right|$ are projection operators on the eigenstates $\left|x_{\alpha n}\right\rangle$ of $X_{\alpha}$ for $\alpha=1,2$. We note that for two coupled systems interacting with a common reservoir [65-67], the same expression (9) will follow. Beyond the one-dimensional baths considered here, determining the MFG state for three-dimensional systems, such as a single spin coupled simultaneously to baths in three dimensions [38], require multibath extensions of (3). It would also be interesting to establish what state the system would take, if it is in contact with two reservoirs at different temperatures.

As an example for Eq. (9) we consider a two qubit system with Hamiltonian $H_{1}+H_{2}+H_{12}^{\text {int }}=\frac{1}{2} \omega_{q}\left(\sigma_{1 z}+\sigma_{2 z}\right)+$ $\lambda_{S}\left(\sigma_{1+} \sigma_{2-}+\sigma_{1-} \sigma_{2+}\right)$ with $\sigma_{n z}=\left|e_{n}\right\rangle\left\langle e_{n}|-| g_{n}\right\rangle\left\langle g_{n}\right|$ and $\sigma_{n-}=\left|g_{n}\right\rangle\left\langle e_{n}\right|=\sigma_{n+}^{\dagger}$ for $n=1,2$ and $\lambda_{S}$ the interqubit coupling. The qubits are each ultrastrongly coupled to a reservoir in the $x$ direction, i.e., $X_{1}=\sigma_{1 x}$ and $X_{2}=\sigma_{2 x}$ with $\sigma_{n x}=\sigma_{n+}+\sigma_{n-}$. The required projection operators are then $P_{n \pm}=\left| \pm_{n}\right\rangle\left\langle \pm_{n}\right|$ with $\left| \pm_{n}\right\rangle=\left(\left|e_{n}\right\rangle \pm\left|g_{n}\right\rangle\right) / \sqrt{2}$. Thus, at ultrastrong coupling, the MFG state of the two qubits is [46],

$$
\rho_{S}=\frac{1}{4}\left[1-\sigma_{1 x} \otimes \sigma_{2 x} \tanh \left(\frac{1}{2} \beta \lambda_{S}\right)\right],
$$

which is independent of $\omega_{q}$. Clearly, while the systemenvironment coupling parameter $\lambda$ does not feature, the state does depend on the interqubit coupling $\lambda_{S}$. For vanishing $\lambda_{S}$ the state loses its energetic coherences, while at high $\lambda_{S}$ these are maximized.

For comparison, the conjectured steady state [63] $\varrho_{S}^{\text {conj }}=$ $\frac{1}{4}\left\{1-\sigma_{1 x} \otimes \sigma_{2 x}\left[\sinh \beta \lambda_{S} /\left(\cosh \beta \omega_{q}+\cosh \beta \lambda_{S}\right)\right]\right\} \quad$ also has tensor product structure, but depends on $\omega_{q}$. It loses this dependence and becomes identical to (10) at high temperatures, $\beta \omega_{q} \ll 1$. Dynamical convergence to $\varrho_{S}^{\text {conj }}$ was numerically evidenced using HEOM [63], but is again consistent with convergence to (10) for the parameters chosen, see figure (ii) in the Supplemental Material [46].

Future research could provide a clearer disambiguation at lower temperatures, however, the HEOM method has its own convergence restrictions that may limit the range of parameters that can be explored. A recent alternative numerical method, TEMPO, is based on time-evolving matrix product operators [68], and can efficiently describe the time evolution of quantum systems coupled to a nonMarkovian harmonic environment. Furthermore, analytical approaches to solve the dynamics when the coupling is no longer weak could be based on reaction coordinate methods [15,16,39,40,69-75].

Conclusion and open questions.-For general quantum systems coupled to a bosonic reservoir two explicit expressions for the MFG state $\rho_{S}$ defined in Eq. (1) have been derived. Results (7) and (3) are valid for any quantum system, be it single qubits, $V$-systems, harmonic oscillators, or others, and make it possible to immediately identify $\rho_{S}$ for a multitude of problems and applications arising in quantum thermodynamics, quantum thermometry, magnetism, quantum biology, and beyond. Taken together, expressions (3) and (7) show evidence of the departure from the textbook Gibbs state, diagonal in $H_{S}$, whenever the 
system-environment coupling is not negligible and the temperature is not large. With increasing coupling, the basis $\left\{P_{n}\right\}$ of the system's interaction operator $X$ becomes increasingly dominant, culminating in the ultrastrong limit where it is the only relevant basis.

To further explore this transition will require finding MFG state expressions for intermediate coupling strengths - a very difficult analytical problem that may first be solved with numerical methods. We highlight that, while it is known that the dynamics of an open quantum system converges to the MFG state in the weak coupling limit [26,52-55], the jury is out for the intermediate and ultrastrong coupling limit. Another open question concerns extensions of the MFG state forms derived here to systems coupled to multiple bosonic reservoirs at the same temperature [38], besides the two qubit example given in Eq. (10). Finally, we expect that extensions to fermionic reservoirs can readily be made, as the derivations leading to (3) and (7) do not centrally depend on the bosonic nature of the reservoir.

We thank Ryoichi Kawai, Steve Barnett, Marco Berritta, Daniel Burgarth, Federico Cerisola, Luis Correa, Giacomo Guarnieri, Simon Horsley, and Stefano Scali for inspiring discussions. J.A. and J.D.C. acknowledge funding from EPSRC (EP/R045577/1), and J.A. thanks the Royal Society for support.

Note added.-A paper has recently been posted [76] that derives an ultrastrong coupling master equation and confirms dynamical convergence to the ultrastrong MFG state (7) derived here.

*j.d.cresser@exeter.ac.uk †janet@qipc.org

[1] H. J. D. Miller, Hamiltonian of mean force for stronglycoupled systems, in Thermodynamics in the Quantum Regime: Fundamental Aspects and New Directions, edited by F. Binder, L. A. Correa, C. Gogolin, J. Anders, and G. Adesso (Springer International Publishing, Cham, 2018), pp. 531549, 10.1007/978-3-319-99046-0_22.

[2] C. Jarzynski, Nonequilibrium work theorem for a system strongly coupled to a thermal environment, J. Stat. Mech. (2004) P09005.

[3] M. Campisi, P. Talkner, and P. Hänggi, Fluctuation Theorem for Arbitrary Open Quantum Systems, Phys. Rev. Lett. 102, 210401 (2009).

[4] M. Campisi, P. Talkner, and P. Hänggi, Thermodynamics and fluctuation theorems for a strongly coupled open quantum system: an exactly solvable case, J. Phys. A 42, 392002 (2009).

[5] M. F. Gelin and M. Thoss, Thermodynamics of a subensemble of a canonical ensemble, Phys. Rev. E 79, 051121 (2009).
[6] S. Hilt, S. Shabbir, J. Anders, and E. Lutz, Landauer's principle in the quantum regime, Phys. Rev. E 83, 030102 (R) (2011).

[7] S. Hilt, B. Thomas, and E. Lutz, Hamiltonian of mean force for damped quantum systems, Phys. Rev. E 84, 031110 (2011).

[8] U. Seifert, First and Second Law of Thermodynamics at Strong Coupling, Phys. Rev. Lett. 116, 020601 (2016).

[9] T. G. Philbin and J. Anders, Thermal energies of classical and quantum damped oscillators coupled to reservoirs, J. Phys. A Math. Theor. 49, 215303 (2016).

[10] C. Jarzynski, Stochastic and Macroscopic Thermodynamics of Strongly Coupled Systems, Phys. Rev. X 7, 011008 (2017).

[11] E. Aurell, On work and heat in time-dependent strong coupling, Entropy 19, 595 (2017).

[12] P. Strasberg and M. Esposito, Stochastic thermodynamics in the strong coupling regime: An unambiguous approach based on coarse graining, Phys. Rev. E 95, 062101 (2017).

[13] H. J. D. Miller and J. Anders, Entropy production and time asymmetry in the presence of strong interactions, Phys. Rev. E 95, 062123 (2017).

[14] E. Aurell, Unified picture of strong-coupling stochastic thermodynamics and time reversals, Phys. Rev. E 97, 042112 (2018).

[15] G. Schaller, J. Cerrillo, G. Engelhardt, and P. Strasberg, Electronic Maxwell demon in the coherent strong-coupling regime, Phys. Rev. B 97, 195104 (2018).

[16] P. Strasberg, G. Schaller, T. L. Schmidt, and M. Esposito, Fermionic reaction coordinates and their application to an autonomous Maxwell demon in the strong-coupling regime, Phys. Rev. B 97, 205405 (2018).

[17] H. Miller and J. Anders, Energy-temperature uncertainty relation in quantum thermodynamics, Nat. Commun. 9, 2203 (2018).

[18] P. Strasberg, Repeated Interactions and Quantum Stochastic Thermodynamics at Strong Coupling, Phys. Rev. Lett. 123, 180604 (2019).

[19] L. A. Correa, M. Perarnau-Llobet, K. V. Hovhannisyan, S. Hernández-Santana, M. Mehboudi, and A. Sanpera, Enhancement of low-temperature thermometry by strong coupling, Phys. Rev. A 96, 062103 (2017).

[20] K. V. Hovhannisyan and L. A. Correa, Measuring the temperature of cold many-body quantum systems, Phys. Rev. B 98, 045101 (2018).

[21] M. Perarnau-Llobet, H. Wilming, A. Riera, R. Gallego, and J. Eisert, Strong Coupling Corrections in Quantum Thermodynamics, Phys. Rev. Lett. 120, 120602 (2018).

[22] W.-M. Huang and W.-M. Zhang, Strong coupling quantum thermodynamics with renormalized hamiltonian and temperature, arXiv:2010.01828v1.

[23] A. Rivas, Strong Coupling Thermodynamics of Open Quantum Systems, Phys. Rev. Lett. 124, 160601 (2020).

[24] J. G. Kirkwood, Statistical mechanics of fluid mixtures, J. Chem. Phys. 3, 300 (1935).

[25] B. Roux, The calculation of the potential of mean force using computer simulations, Comput. Phys. Comms. 91, 275 (1995).

[26] A. Purkayastha, G. Guarnieri, M. T. Mitchison, R. Filip, and J. Goold, Tunable phonon-induced steady-state coherence 
in a double-quantum-dot charge qubit, npj Quantum Inf. 6, 27 (2020).

[27] A. Streltsov, G. Adesso, and M. B. Plenio, Colloquium: Quantum coherence as a resource, Rev. Mod. Phys. 89, 041003 (2017).

[28] R. Uzdin, A. Levy, and R. Kosloff, Equivalence of Quantum Heat Machines, and Quantum-Thermodynamic Signatures, Phys. Rev. X 5, 031044 (2015).

[29] P. Kammerlander and J. Anders, Coherence and measurement in quantum thermodynamics, Sci. Rep. 6, 22174 (2016).

[30] G. Francica, F. C. Binder, G. Guarnieri, M. T. Mitchison, J. Goold, and F. Plastina, Quantum Coherence and Ergotropy, Phys. Rev. Lett. 125, 180603 (2020).

[31] A. Messinger, A. Ritboon, F. Crimin, S. Croke, and S. M. Barnett, Coherence and catalysis in the Jaynes-Cummings model, New J. Phys. 22, 043008 (2020).

[32] K. Hammam, Y. Hassouni, R. Fazio, and G. Manzano, Optimizing autonomous thermal machines powered by energetic coherence, New J. Phys. 23, 043024 (2021).

[33] S. Lloyd, Quantum coherence in biological systems, J. Phys. 302, 012037 (2011).

[34] N. Lambert, Y.-N. Chen, Y.-C. Cheng, C.-M. Li, G.-Y. Chen, and F. Nori, Quantum biology, Nat. Phys. 9, 10 (2013).

[35] J. Jeske, D. J. Ing, M. B. Plenio, S. F. Huelga, and J. H. Cole, Bloch-Redfield equations for modeling light-harvesting complexes, J. Chem. Phys. 142, 064104 (2015).

[36] A. Dodin, T. V. Tscherbul, and P. Brumer, Quantum dynamics of incoherently driven V-type systems: Analytic solutions beyond the secular approximation, J. Chem. Phys. 144, 244108 (2016).

[37] A. Dodin, T. V. Tscherbul, R. Alicki, A. Vutha, and P. Brumer, Secular versus nonsecular Redfield dynamics and Fano coherences in incoherent excitation: An experimental proposal, Phys. Rev. A 97, 013421 (2018).

[38] J. Anders, C. R. J. Sait, and S. A. R. Horsley, Quantum brownian motion for magnets, arXiv:2009.00600.

[39] D. Newman, F. Mintert, and A. Nazir, Performance of a quantum heat engine at strong reservoir coupling, Phys. Rev. E 95, 032139 (2017).

[40] A. Nazir and G. Schaller, The reaction coordinate mapping in quantum thermodynamics, in Thermodynamics in the Quantum Regime: Fundamental Aspects and New Directions, edited by F. Binder, L. A. Correa, C. Gogolin, J. Anders, and G. Adesso (Springer International Publishing, Cham, 2018), pp. 551-577, 10.1007/978-3-319-99046-0_23.

[41] For $H_{S}$ bounded from below, $H_{S R}$ will clearly also be bounded from below. However, if one took instead the form $H_{S R}=H_{S}+H_{R}+\lambda X B[40,42]$ then energies unbounded from below can arise at strong coupling for systems of infinite dimension. Care must be taken when applying the results derived here in such situations.

[42] G. W. Ford and R. F. O'Connell, The rotating wave approximation (RWA) of quantum optics: serious defect, Physica (Amsterdam) 243A, 377 (1997).

[43] J. Wu, F. Liu, Y. Shen, J. Cao, and R. J. Silbey, Efficient energy transfer in light-harvesting systems, I: optimal temperature, reorganization energy and spatial-temporal correlations, New J. Phys. 12, 105012 (2010).
[44] G. Ritschel, J. Roden, W. T. Strunz, and A. Eisfeld, An efficient method to calculate excitation energy transfer in light-harvesting systems: application to the FennaMatthews-Olson complex, New J. Phys. 13, 113034 (2011).

[45] A. Fruchtman, N. Lambert, and E. M. Gauger, When do perturbative approaches accurately capture the dynamics of complex quantum systems? Sci. Rep. 6, 28204 (2016).

[46] See Supplemental Material at http://link.aps.org/ supplemental/10.1103/PhysRevLett.127.250601 for details of calculations and results for: the high temperature limit via a factorization approximation, the Kubo expansion for weak coupling at arbitrary temperature, applications of the weak coupling result, the derivation of the ultrastrong limit expression, and applications of the ultrastrong coupling result. Some of the derivations build on previous work reported in Refs. [47-51].

[47] M. Suzuki, On the convergence of exponential operatorsthe Zassenhaus formula, $\mathrm{BCH}$ formula and systematic approximants, Commun. Math. Phys. 57, 193 (1977).

[48] E. Geva, E. Rosenman, and D. Tannor, On the second-order corrections to the quantum canonical equilibrium density matrix, J. Chem. Phys. 113, 1380 (2000).

[49] R. Kubo, Statistical-mechanical theory of irreversible processes. I. General theory and simple applications to magnetic and conduction problems, J. Phys. Soc. Jpn. 12, 570 (1957).

[50] G. Guarnieri, M. Kolár, and R. Filip, Steady-State Coherences by Composite System-Bath Interactions, Phys. Rev. Lett. 121, 070401 (2018).

[51] D. Burgarth, P. Facchi, H. Nakazato, S. Pascazio, and K. Yuasa, Kolmogorov-Arnold-Moser Stability for Conserved Quantities in Finite-Dimensional Quantum Systems, Phys. Rev. Lett. 126, 150401 (2021).

[52] T. Mori and S. Miyashita, Dynamics of the density matrix in contact with a thermal bath and the quantum master equation, J. Phys. Soc. Jpn. 77, 124005 (2008).

[53] C. H. Fleming and N. I. Cummings, Accuracy of perturbative master equations, Phys. Rev. E 83, 031117 (2011).

[54] J. Thingna, J.-S. Wang, and P. Hänggi, Generalized Gibbs state with modified Redfield solution: Exact agreement up to second order, J. Chem. Phys. 136, 194110 (2012).

[55] Y. Subaş1, C. H. Fleming, J. M. Taylor, and B. L. Hu, Equilibrium states of open quantum systems in the strong coupling regime, Phys. Rev. E 86, 061132 (2012).

[56] G. S. Agarwal and S. Menon, Quantum interferences and the question of thermodynamic equilibrium, Phys. Rev. A 63, 023818 (2001).

[57] T. V. Tscherbul and P. Brumer, Long-Lived Quasistationary Coherences in a $V$-type System Driven by Incoherent Light, Phys. Rev. Lett. 113, 113601 (2014).

[58] D. Burgarth, P. Facchi, H. Nakazato, S. Pascazio, and K. Yuasa, Generalized adiabatic theorem and strong-coupling limits, Quantum 3, 152 (2019).

[59] B. Misra and E. C. G. Sudarshan, The Zeno's paradox in quantum theory, J. Math. Phys. (N.Y.) 18, 756 (1977).

[60] P. Facchi, Quantum zeno effect, adiabaticity and dynamical superselection rules, in Fundamental Aspects of Quantum Physics, edited by L. Accardi and S. Tasaki (World Scientific, Singapore, 2003), pp. 197-221.

[61] P. Facchi and S. Pascazio, Three different manifestations of the quantum Zeno effect, in Irreversible Quantum 
Dynamics, edited by F. Benatti and R. Floreanini (Springer, Berlin, Heidelberg, 2003), pp. 141-156.

[62] We note that, mathematically, in the ultrastrong limit, the Gibbs state $\tau_{S}$ is not recovered in the high temperature limit $(\beta \rightarrow 0)$, due to issues of the order of limits. However, physically a situation where a quantum system couples ultrastrongly to a reservoir at ultrahigh temperatures, is not realistic, so we leave this mathematical issue unresolved here.

[63] K. Goyal and R. Kawai, Steady state thermodynamics of two qubits strongly coupled to bosonic environments, Phys. Rev. Research 1, 033018 (2019).

[64] P. L. Orman and R. Kawai, A qubit strongly interacting with a bosonic environment: Geometry of thermal states, arXiv: 2010.09201v1.

[65] P. P. Orth, D. Roosen, W. Hofstetter, and K. Le Hur, Dynamics, synchronization, and quantum phase transitions of two dissipative spins, Phys. Rev. B 82, 144423 (2010).

[66] F. Benatti, R. Floreanini, and U. Marzolino, Entangling two unequal atoms through a common bath, Phys. Rev. A 81, 012105 (2010).

[67] T. Deng, Y. Yan, L. Chen, and Y. Zhao, Dynamics of the two-spin spin-boson model with a common bath, J. Chem. Phys. 144, 144102 (2016).

[68] A. Strathearn, P. Kirton, D. Kilda, J. Keeling, and B. Lovett, Efficient non-Markovian quantum dynamics using timeevolving matrix product operators, Nat. Commun. 9, 3322 (2018).
[69] R. S. Burkey and C. D. Cantrell, Discretization in the quasicontinuum, JOSA B 1, 169 (1984).

[70] A. Garg, J. N. Onuchic, and V. Ambegaokar, Effect of friction on electron transfer in biomolecules, J. Chem. Phys. 83, 4491 (1985).

[71] J. Iles-Smith, N. Lambert, and A. Nazir, Environmental dynamics, correlations, and the emergence of noncanonical equilibrium states in open quantum systems, Phys. Rev. A 90, 032114 (2014).

[72] J. Iles-Smith, A. G. Dijkstra, N. Lambert, and A. Nazir, Energy transfer in structured and unstructured environments: Master equations beyond the Born-Markov approximations, J. Chem. Phys. 144, 044110 (2016).

[73] P. Strasberg, G. Schaller, N. Lambert, and T. Brandes, Nonequilibrium thermodynamics in the strong coupling and non-Markovian regime based on a reaction coordinate mapping, New J. Phys. 18, 073007 (2016).

[74] S. Restrepo, J. Cerrillo, P. Strasberg, and G. Schaller, From quantum heat engines to laser cooling: Floquet theory beyond the Born-Markov approximation, New J. Phys. 20, 053063 (2018).

[75] N. Anto-Sztrikacs and D. Segal, Strong coupling effects in quantum thermal transport with the reaction coordinate method, New J. Phys. 23, 063036 (2021).

[76] A. Trushechkin, Quantum master equations and steady states for the ultrastrong-coupling limit and the strongdecoherence limit, arXiv:2109.01888v1. 